\title{
LINC00628 is differentially expressed between lung adenocarcinoma and squamous cell carcinoma and is associated with the prognosis of NSCLC
}

\author{
TINGTING LIU ${ }^{1}$, SHUANGSHUANG XU ${ }^{2}$ and XIAOXIN LIU ${ }^{3}$ \\ ${ }^{1}$ Health Management Center; ${ }^{2}$ Department of Obstetrics, Weifang People's Hospital, Weifang, Shandong 261041; \\ ${ }^{3}$ Emergency Department, Weifang People's Hospital Brain Hospital, Weifang, Shandong 100191, P.R. China
}

Received August 4, 2021; Accepted December 1, 2021

DOI: $10.3892 / \mathrm{ol} .2021 .13173$

\begin{abstract}
Non-small cell lung cancer (NSCLC) remains the most frequent malignancy worldwide, and lung adenocarcinoma (LUAD) and lung squamous cell carcinoma (LUSC) represent two major subtypes. LINC00628 has been demonstrated to promote LUAD progression; however, its clinical role in NSCLC remains elusive. The aim of the present study was to analyze the expression of long intergenic non-protein coding RNA 628 (LINC00628) in NSCLC, including in the LUAD and LUSC subtypes. In addition, its roles in NSCLC development and prognosis were also examined. Data from The Cancer Genome Atlas (TCGA) database were first used to assess the expression and prognostic potential in both LUAD and LUSC, then LINC00628 expression in 128 NSCLC tissues was measured using reverse transcription-quantitative PCR. A receiver operating characteristic curve was used to assess the ability of LINC00628 to discriminate between patients with LUAD and LUSC. Kaplan-Meier curves were used to analyze the relationship between LINC00628 expression and the overall survival of patients. Cox regression analysis was used to explore the potential prognostic factors that might be independently associated with NSCLC overall survival. Both in silico and tissue analysis data indicated that the expression of LINC00628 was significantly upregulated in NSCLC tissue compared with matched normal controls $(\mathrm{P}<0.001)$. LINC00628 expression levels were also significantly higher in LUAD cases than in patients with LUSC $(\mathrm{P}<0.001)$. In addition, LINC00628 could discriminate LUAD from LUSC cases. The expression of LINC00628 was significantly associated with tumor size $(\mathrm{P}=0.013)$, histological type $(\mathrm{P}=0.009)$, lymph node metastasis $(\mathrm{P}=0.021)$ and $\mathrm{TNM}$ stage $(\mathrm{P}=0.008)$.
\end{abstract}

Correspondence to: Dr Xiaoxin Liu, Emergency Department, Weifang People's Hospital Brain Hospital, 553 Dongfeng West Street, Weicheng, Weifang, Shandong 100191, P.R. China E-mail: liuxiaoxin_wfrmnk@163.com

Key words: LINC00628, lung adenocarcinoma, lung squamous cell carcinoma, prognosis, overall survival
Survival analysis based on data from both TCGA and patients included in the present study identified an association between LINC00628 and overall survival in LUAD, but this relationship was not observed in LUSC for TCGA data. Cox regression analysis demonstrated that high LINC00628 expression was associated with poor overall survival in patients with LUAD $(\mathrm{P}=0.001)$, but not in patients with LUSC $(\mathrm{P}=0.088)$. In conclusion, LINC00628 expression was upregulated in NSCLC and associated with patient prognosis. Patients with LUAD had higher LINC00628 expression levels than those with LUSC, and increased LINC00628 served as an independent prognostic factor in LUAD, but not LUSC.

\section{Introduction}

Lung cancer is one of the most commonly diagnosed malignancies, and accounts for 1.6 million deaths annually worldwide; in addition, non-small cell lung cancer (NSCLC) makes up the majority of lung cancer cases $(1,2)$. The prognosis of patients with NSCLC is generally poor, with a 5-year overall survival rate of $<18 \%$ (3), and even lower for patients in advanced stages $(0-10 \%)(4)$. Different subtypes of NSCLC are associated with different molecular biological characteristics (5). The main histological subtypes of NSCLC include lung adenocarcinoma (LUAD) and lung squamous cell carcinoma (LUSC) (6). There is a significant difference in the clinical treatment between the LUAD and LUSC subtypes. For instance, patients with LUSC experience a longer overall survival time than those with LUAD after treatment with ipilimumab (7). In addition, treatment with gefitinib, which targets EGFR kinase mutated cases, is more suitable for patients with LUAD (8). Therefore, the development of effective molecular diagnostic biomarkers and potential molecules for the identification and treatment of the LUAD and LUSC subtypes is of significant interest.

Long non-coding RNA (lncRNA) is a type of non-coding transcript $>200$ nucleotides in length (9). lncRNA molecules have emerged as key regulators in tumor development and progression (10-12). For example, Wei et al (13) suggested that BCAR4 contributed to glioma progression by enhancing cell proliferation and activating the EGFR/PI3K/AKT pathway, which may represent a new target for the treatment and prognosis of patients with glioma. Upregulation of the lncRNA 
HOTTIP has also been recognized as a marker of poor survival in patients with renal cell carcinoma (14).

Long intergenic non-protein coding RNA 628 (LINC00628) is an IncRNA that has been reported to suppress the growth and metastasis of breast cancer and promote the apoptosis of breast cancer cells in previous studies $(15,16)$. Zhang et al $(17)$ also suggested that downregulation of LINC00628 aggravated the progression of colorectal cancer by inhibiting p57 expression. Recent studies have described the aberrant expression of mRNAs, circRNAs and lncRNAs that could distinguish between LUAD and LUSC (18-20), indicating the importance of analyzing functional molecules in different subtypes of NSCLC. Xu et al (20) demonstrated that the expression levels of LINC00628 were upregulated in LUAD tissue, which promoted LUAD cell proliferation, migration and invasion. However, the expression levels and clinical significance of LINC00628 in other subtypes of NSCLC remain poorly understood.

The aim of the present study was to analyze the expression levels of LINC00628 in NSCLC and normal tissue, as well as in the LUAD and LUSC subtypes. Moreover, the ability of LINC00628 to discriminate between patients with LUAD and those with LUSC was also evaluated. Lastly, the clinical significance of LINC00628 in patient prognosis was also analyzed in patients with NSCLC.

\section{Materials and methods}

Patients and tissue collection. Tissue samples were collected from 128 patients with NSCLC, including 73 patients with LUAD and 55 patients with LUSC, who underwent curative resection between February 2015 and October 2019 at Weifang People's Hospital (Weifang, Shandong, China). NSCLC and adjacent normal tissue samples (located $3 \mathrm{~cm}$ from the edge of the tumors) were collected from the patients. All tissue samples were verified by histopathological examination and stored in liquid nitrogen for further use. The inclusion criteria were as follows: i) The patients were diagnosed with NSCLC by pathological examination; ii) had not received any tumor therapy before surgery; and iii) had a complete clinicopathological record. The following patients were excluded from the study: i) Patients $<18$ years or $>80$ years; ii) pregnant or lactating patients; iii) patients with autoimmune diseases or other malignancies; iv) patients who had received any anti-tumor treatment. Follow-up surveys after the surgery were conducted by telephone or via outpatient visits. Survival was recorded for all patients. The protocols used for tissue collection and analysis were approved by The Ethics Committee of Weifang People's Hospital (approval no. 0014647) and adhered with its guidelines. Written informed consent was obtained from the patients or their family members (for patients who had no ability to read and/or write) prior to sample collection.

Bioinformatics analysis. In the present study, starBase v3.0 (http://starbase.sysu.edu.cn/index.php) was used to analyze The Cancer Genome Atlas (TCGA) datasets in order to compare the expression levels of LINC00628 in LUAD, LUSC and matched normal tissues from patients with cancer. In addition, Gene Expression Profiling Interactive Analysis (http://gepia. cancer-pku.cn/index.html) was used to generate the survival curves of patients with LUAD and LUSC based on the expression of LINC00628, as well as to evaluate the association between LINC00628 and overall survival prognosis.

RNA extraction. TRIzol ${ }^{\circledR}$ reagent (Invitrogen; Thermo Fisher Scientific, Inc.) was used to extract total RNA from tissue samples. NanoDrop ${ }^{\circledR} 2000$ (Thermo Fisher Scientific, Inc.) was used to measure the purity and concentration of the extracted RNA. cDNA was then reverse-transcribed from RNA using the RevertAid First Strand cDNA Synthesis Kit (Thermo Fisher Scientific, Inc.) at $42^{\circ} \mathrm{C}$ for $30 \mathrm{~min}$ followed by $85^{\circ} \mathrm{C}$ for $5 \mathrm{sec}$.

Reverse transcription-quantitative PCR (RT-qPCR). The expression levels of LINC00628 were measured using RT-qPCR, which was carried out using a SYBR Green PCR Kit (Bio-Rad laboratories, Inc.) on an Applied Biosystems 7900 Real-Time PCR system (Applied Biosystems; Thermo Fisher Scientific, Inc.). The thermocycling conditions were: i) Initial denaturation for $10 \mathrm{~min}$ at $95^{\circ} \mathrm{C}$; ii) 40 cycles of denaturation for $30 \mathrm{sec}$ at $95^{\circ} \mathrm{C}$, annealing for $20 \mathrm{sec}$ at $58^{\circ} \mathrm{C}$ and extension for $30 \mathrm{sec}$ at $72^{\circ} \mathrm{C}$; and iii) final extension for $10 \mathrm{~min}$ at $72^{\circ} \mathrm{C}$. GAPDH was used as an endogenous control for LINC00628. The primer sequences were as follows: LINC00628 forward, 5'-ACTCCGCCTGGATGGGAATA-3' and reverse, 5'-CAGGACTTGGCCCACCTATC-3'; GAPDH forward, 5'-CAAGGTCATCCATGACAACTTTG-3' and reverse, 5'-GTCCACCACCCTGTTGCTGTAG-3'. All protocols were performed following the manufacturer's instructions. The expression levels of LINC00268 were calculated using the $2^{-\Delta \Delta C q}$ method (21) and normalized to those of GAPDH.

Statistical analysis. All data are shown as the mean \pm SD. SPSS 22.0 (IBM Corp.) and GraphPad Prism 7.0 software (GraphPad Software, Inc.) were used to perform the statistical analysis. Paired Student's t-tests were used to compare the differences in LINC00628 expression between tumor tissue samples (NSCLC, LUAD or LUSC) and adjacent healthy tissue controls. Unpaired Student's t-test was used to analyze the differences in LINC00628 expression between LUAD and LUSC cases. The $\chi^{2}$ test was used to assess the relationship between LINC00628 and the clinicopathological features of patients with NSCLC. The ability of LINC00628 to discriminate between patients with LUAD and LUSC was assessed using a receiver operating characteristic (ROC) curve. In this analysis, the expression of LINC00628 in LUAD and LUSC was used to construct ROC curve, and the area under the curve (AUC) was calculated to indicate the accuracy of LINC00628 in distinguishing LUAD and LUSC. The diagnostic sensitivity and specificity were obtained at the optimal cut-off value point, which was the value when the sum of the sensitivity and specificity of the ROC curve was the highest. The relationship between LINC00628 expression and the overall survival of patients with NSCLC, LUAD or LUSC was analyzed using Kaplan-Meier curves. The patients were divided into low- and high-expression groups according to the median of the group. Univariate and multivariate Cox regression analysis was used to examine the prognostic value of LINC00628 in patients with NSCLC. Each experiment was repeated at least three times. $\mathrm{P}<0.05$ was considered to indicate a statistically significant difference. 
A

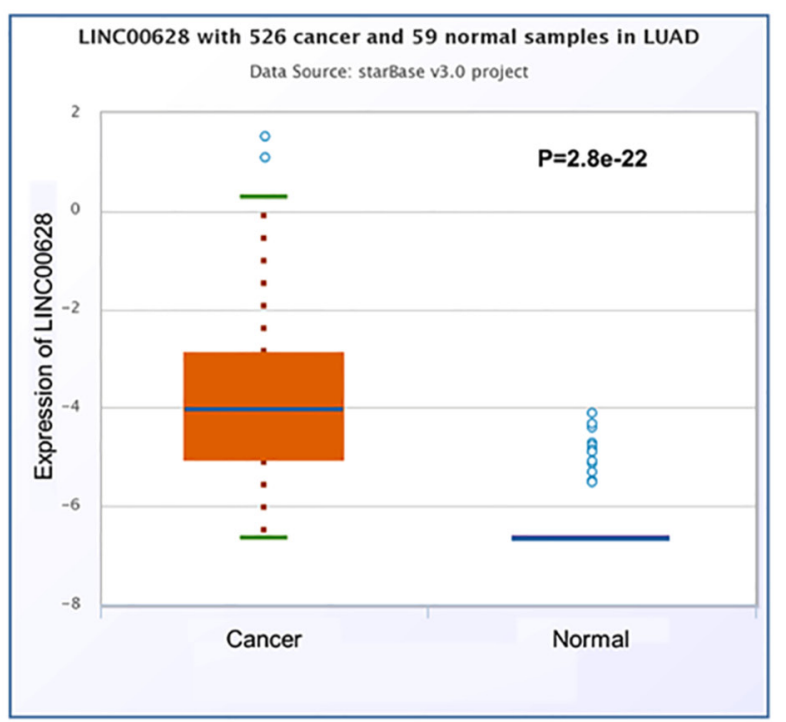

C

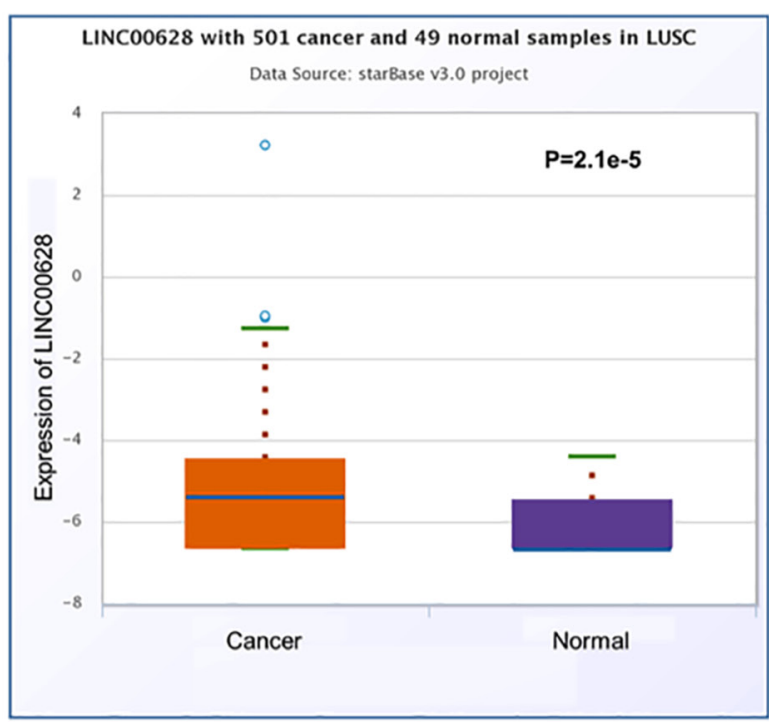

B

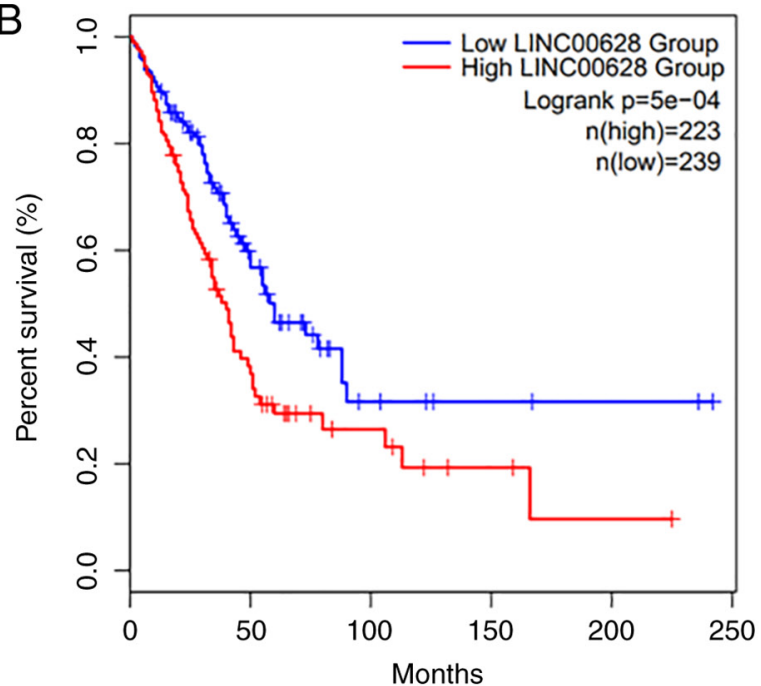

D

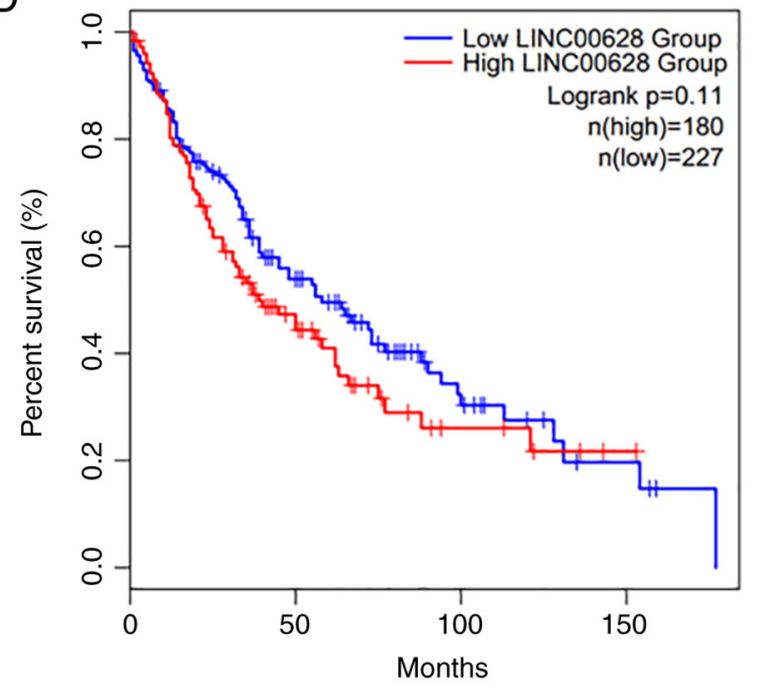

Figure 1. Bioinformatics analysis of LINC00628 expression and its association with overall survival in non-small cell lung cancer. (A) Expression of LINC00628 in LUAD and normal samples (mean, 0.11 vs. 0.02 , respectively; $\mathrm{P}=2.8 \times 10^{-22}$ ). (B) Relationship between LINC00628 expression and overall survival of patients with LUAD (log-rank $\mathrm{P}=5 \times 10^{-4} ; \mathrm{HR}=1.7$ ). (C) Expression of LINC00628 in LUSC and normal samples (mean, 0.06 vs. 0.02 , respectively; $\mathrm{P}=2.1 \times 10^{-5}$ ). (D) Relationship between LINC00628 expression and overall survival of patients with LUSC (log-rank $\mathrm{P}=0.11 ; \mathrm{HR}=1.3$ ). LINC00628, long intergenic non-protein coding RNA 628; LUAD, lung adenocarcinoma; LUSC, lung squamous cell carcinoma; HR, hazard ratio.

\section{Results}

LINC00628 expression is associated with the overall survival of patients with NSCLC. Bioinformatics analysis of TCGA datasets suggested that the expression levels of LINC00628 were significantly upregulated in LUAD $(n=526)$ compared with adjacent normal tissues $(\mathrm{n}=59)($ Fig. $1 \mathrm{~A}$; mean, 0.11 vs. 0.02 , respectively; $\mathrm{P}=2.8 \times 10^{-22}$ ). The Kaplan-Meier curves generated using TCGA data also indicated that patients with LUAD and high LINC00628 expression experienced worse overall survival compared with those with low LINC00628 expression [Fig. 1B; log-rank $\mathrm{P}=5 \times 10^{-4}$; hazard ratio $(\mathrm{HR}=1.7)]$. Moreover, the expression levels of LINC00628 were significantly increased in LUSC $(n=501)$ compared with adjacent normal tissues ( $\mathrm{n}=59$ ) (Fig. 1C; mean, 0.06 vs. 0.02 , respectively; $\mathrm{P}=2.1 \times 10^{-5}$ ). Kaplan-Meier curves for patients with LUSC indicated that there was no significant difference in overall survival between patients with high or low LINC00628 expression (Fig. 1D; log-rank $\mathrm{P}=0.11$; HR=1.33).

LINC00628 expression is dysregulated patients with LUAD and LUSC. The expression levels of LINC00628 were significantly upregulated NSCLC tumor compared with the adjacent normal tissue samples (Fig. 2A; $\mathrm{n}=128 ; \mathrm{P}<0.001$ ). In addition, the expression of LINC00628 was also significantly increased in LUAD $(n=73)$ and LUSC $(n=55)$ tumor tissue compared with the adjacent normal tissue controls (Fig. 2B, $\mathrm{P}<0.001$ ). Moreover, LINC00628 expression was significantly increased in patients with LUAD compared with those with LUSC (Fig. 2C, P<0.001). The expression of LINC00628 in LUAD and LUSC was used to construct an ROC curve, and the AUC was calculated as a measure of the accuracy of LINC00628 in discriminating between LUAD and LUSC. The ROC curve results revealed that the AUC of the curve was 0.861 , indicating 
A
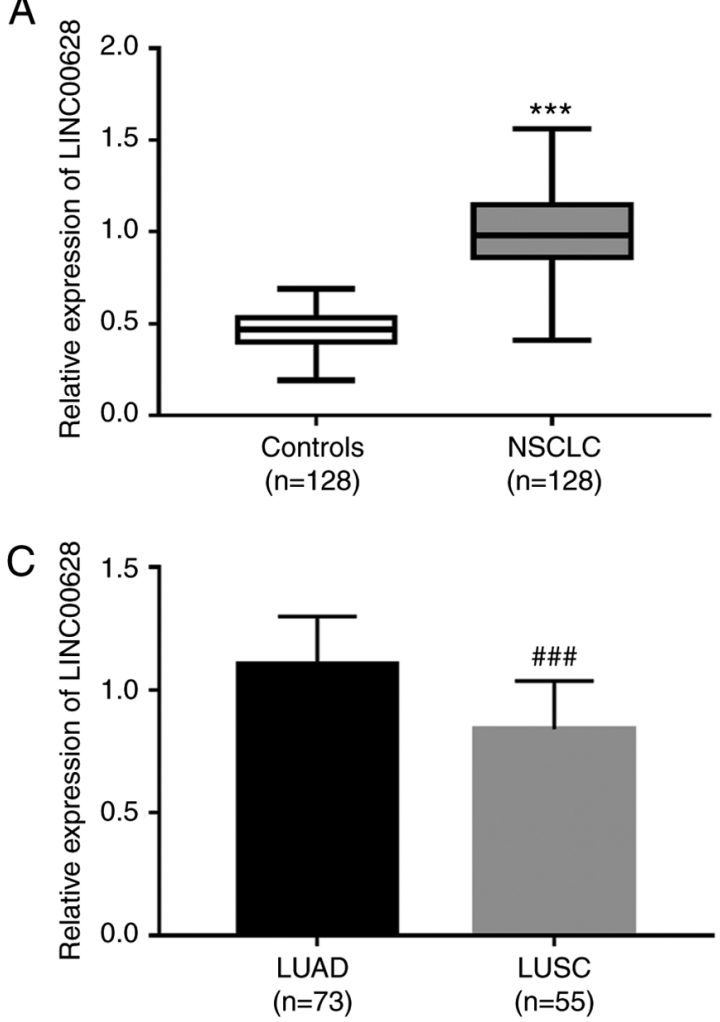

$\mathrm{B}$

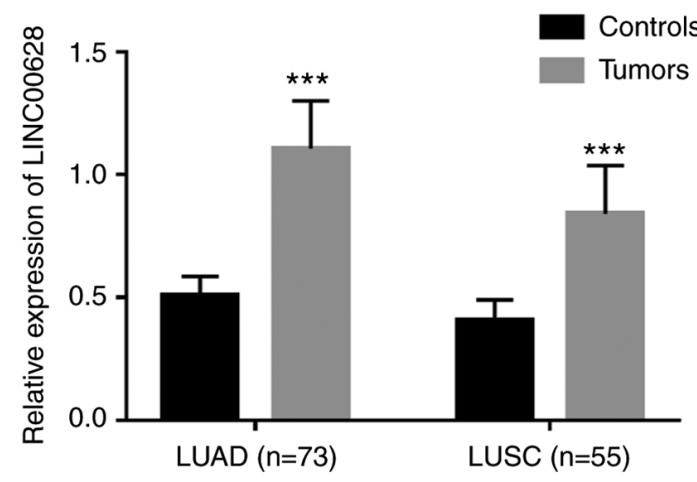

D

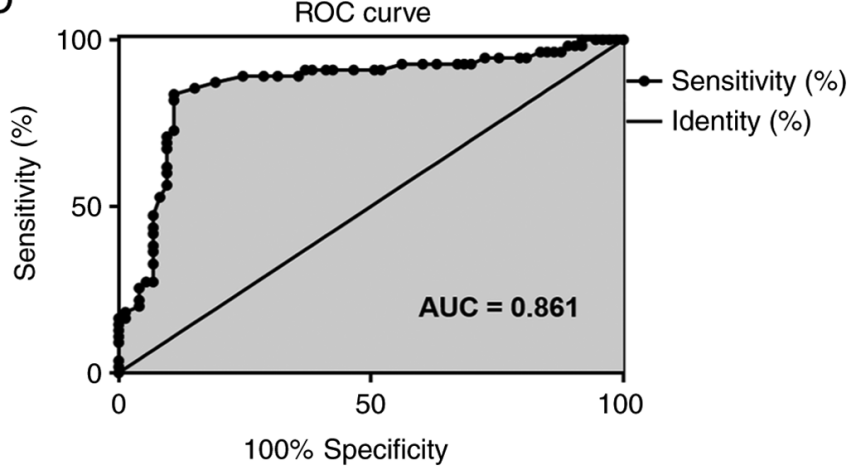

Figure 2. Upregulation of LINC00628 in LUAD and LUSC tumor tissue. (A) Relative expression of LINC00628 in NSCLC tumor and adjacent normal tissue samples (mean, 0.99 vs. 0.46, respectively; ${ }^{* * *} \mathrm{P}<0.001$ vs. Controls). (B) Relative expression of LINC00628 in LUAD and LUSC tumors, compared with adjacent normal tissue samples (LUAD, mean, 1.11 vs. 0.51 , respectively, ${ }^{* * * *} \mathrm{P}<0.001$ vs. Controls; LUSC, mean, 0.84 vs. 0.41 , respectively, ${ }^{* * * *} \mathrm{P}<0.001$ vs. Controls). (C) Relative expression of LINC00628 in patients with LUAD compared with patients with LUSC (mean, 1.11 vs. 0.84, respectively; $\mathrm{P}<0.001$ ). \#\# $\mathrm{P}<0$. vs. LUAD. (D) LINC00628 expression can discriminate between LUAD and LUSC (AUC=0.861). LINC00628, long intergenic non-protein coding RNA 628; LUAD, lung adenocarcinoma; LUSC, lung squamous cell carcinoma; NSCLC, non-small cell lung cancer; AUC, area under the curve.

A NSCLC

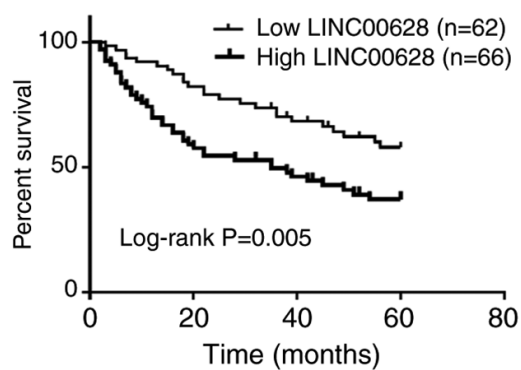

B

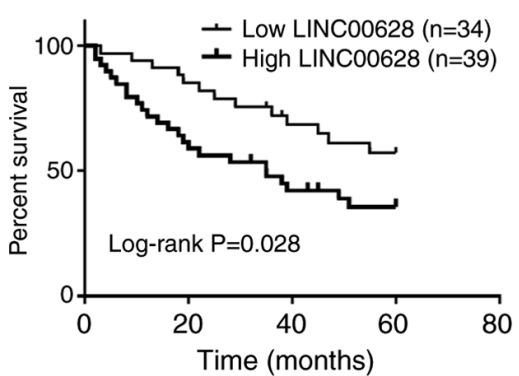

C

LUSC

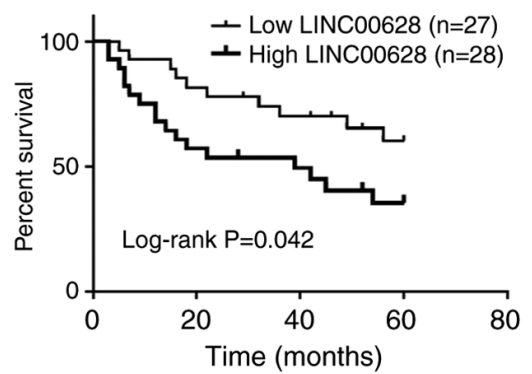

Figure 3. Association between LINC00628 expression and overall survival in patients with NSCLC. (A) High LINC00628 is associated with worse overall survival in all patients with NSCLC (log-rank $\mathrm{P}=0.005$ ). (B and $\mathrm{C}$ ) High LINC00628 expression was associated with worse overall survival in (B) patients with LUAD (log-rank $\mathrm{P}=0.028$ ) and $(\mathrm{C})$ patients with LUSC (log-rank P=0.042). LINC00628, long intergenic non-protein coding RNA 628; LUAD, lung adenocarcinoma; LUSC, lung squamous cell carcinoma; NSCLC, non-small cell lung cancer.

that LINC00628 could accurately distinguish LUAD from LUSC. At the optimal cut-off value of 0.955 , the sensitivity was $83.64 \%$, and the specificity was $89.04 \%$ (Fig. 2D).

Relationship between LINC00628 and the clinicopathological features of patients with NSCLC. As shown in Table I, the expression of LINC00628 was significantly associated with tumor size $(\mathrm{P}=0.013)$, histological type $(\mathrm{P}=0.009)$, lymph node metastasis $(\mathrm{P}=0.021)$ and TNM stage $(\mathrm{P}=0.008)$. However, there were no significant associations between LINC00628 expression and age, sex and smoking status (all $\mathrm{P}>0.05$ ). These findings suggested that LINC00628 may be involved in the development of NSCLC.

High LINC00628 is associated poor overall survival in patients with NSCLC. Kaplan-Meier survival curves and multivariate Cox regression analyses were used to evaluate the prognostic value of LINC00628 for patients with NSCLC. As shown in Fig. 3A, high LINC00628 expression was associated with worse overall survival in patients with NSCLC (log-rank 
Table I. Relationship between LINC00628 expression and the clinicopathological characteristics of patients with non-small cell lung cancer.

\begin{tabular}{|c|c|c|c|c|}
\hline \multirow[b]{2}{*}{ Variables } & \multirow[b]{2}{*}{$\begin{array}{c}\text { Total } \\
(n=128)\end{array}$} & \multicolumn{2}{|c|}{$\begin{array}{c}\text { LINC00628 } \\
\text { expression }\end{array}$} & \multirow[b]{2}{*}{ P-value ${ }^{a}$} \\
\hline & & $\begin{array}{c}\text { Low } \\
(n=62)\end{array}$ & $\begin{array}{l}\text { High } \\
(n=66)\end{array}$ & \\
\hline Age, years & & & & 0.656 \\
\hline$\leq 60$ & 45 & 23 & 22 & \\
\hline$>60$ & 83 & 39 & 44 & \\
\hline Sex & & & & 0.809 \\
\hline Female & 53 & 25 & 28 & \\
\hline Male & 75 & 37 & 38 & \\
\hline Smoking & & & & 0.633 \\
\hline No & 53 & 27 & 26 & \\
\hline Yes & 75 & 35 & 40 & \\
\hline Tumor size, cm & & & & 0.013 \\
\hline$\leq 3$ & 66 & 39 & 27 & \\
\hline$>3$ & 62 & 23 & 39 & \\
\hline Histological type & & & & 0.009 \\
\hline LUAD & 73 & 28 & 45 & \\
\hline LUSC & 55 & 34 & 21 & \\
\hline Lymph node metastasis & & & & 0.021 \\
\hline Negative & 65 & 38 & 27 & \\
\hline Positive & 63 & 24 & 39 & \\
\hline TNM stage & & & & 0.008 \\
\hline I-II & 59 & 36 & 23 & \\
\hline III-IV & 69 & 26 & 43 & \\
\hline
\end{tabular}

aHigh vs. low groups. LINC00628, long intergenic non-protein coding RNA 628; LUAD, lung adenocarcinoma; LUSC, lung squamous cell carcinoma; TNM, tumor-node-metastasis.

$\mathrm{P}=0.005)$. Cox regression analysis indicated that lymph node metastasis $(\mathrm{HR}=1.508 ; 95 \% \mathrm{CI}, 1.085-2.167 ; \mathrm{P}=0.047)$, TNM stage $(\mathrm{HR}=2.541 ; 95 \% \mathrm{CI}, 1.883-3.496 ; \mathrm{P}=0.003)$ and LINC00628 (HR=2.887; 95\% CI, 1.973-4.243; $\mathrm{P}=0.002)$ were associated with survival of patients with NSCLC, and TNM stage $(\mathrm{HR}=2.139 ; 95 \% \mathrm{CI}, 1.527-2.896 ; \mathrm{P}=0.008)$ and LINC00628 expression (HR=2.161, 95\% CI, 1.452-3.051; $\mathrm{P}=0.005)$ were confirmed as two independent prognostic factors (Table II).

Furthermore, the prognostic value of LINC00628 in patients with LUAD and LUSC were also analyzed. In LUAD tumor tissue samples, high LINC00628 was associated with worse overall survival (Fig. 3B; log-rank $\mathrm{P}=0.028$ ). Univariate Cox regression analysis results demonstrated the relationship of lymph node metastasis, TNM stage and LINC00628 expression with overall survival of patients with LUAD $(\mathrm{P}<0.05)$, and the results from multivariate Cox regression analysis also indicated that LINC00628 expression $(\mathrm{HR}=2.437 ; 95 \% \mathrm{CI}=1.551-3.496 ; \mathrm{P}=0.001)$, lymph node metastasis $(\mathrm{HR}=1.532$; 95\% CI, 1.035-2.159; $\mathrm{P}=0.047)$, TNM
Table II. Cox regression analysis in patients with non-small cell lung cancer.

A, Univariate analysis

\begin{tabular}{lccc}
\hline Variables & HR & $95 \%$ CI & P-value \\
\hline Age & 1.139 & $0.831-1.556$ & 0.293 \\
Sex & 1.214 & $0.794-1.809$ & 0.302 \\
Smoking & 1.148 & $0.851-1.667$ & 0.227 \\
Tumor size & 1.409 & $0.965-1.973$ & 0.089 \\
Histological type & 1.132 & $0.921-1.480$ & 0.298 \\
Lymph node metastasis & 1.508 & $1.085-2.167$ & 0.047 \\
TNM stage & 2.541 & $1.883-3.496$ & 0.003 \\
LINC00628 expression & 2.887 & $1.973-4.243$ & 0.002 \\
\hline
\end{tabular}

$\mathrm{B}$, Multivariate analysis

\begin{tabular}{lccc}
\hline Variables & HR & $95 \%$ CI & P-value \\
\hline Age & 1.115 & $0.821-1.435$ & 0.334 \\
Sex & 1.261 & $0.854-1.896$ & 0.289 \\
Smoking & 1.124 & $0.864-1.593$ & 0.255 \\
Tumor size & 1.313 & $0.942-1.574$ & 0.192 \\
Histological type & 1.102 & $0.929-1.382$ & 0.283 \\
Lymph node metastasis & 1.415 & $0.993-1.991$ & 0.066 \\
TNM stage & 2.139 & $1.527-2.896$ & 0.008 \\
LINC00628 expression & 2.161 & $1.452-3.051$ & 0.005 \\
\hline
\end{tabular}

LINC00628, long intergenic non-protein coding RNA 628; TNM, tumor-node-metastasis; HR, hazard ratio; CI, confidence interval.

stage $(\mathrm{HR}=2.222 ; 95 \% \mathrm{CI}, 1.533-2.935 ; \mathrm{P}=0.006)$ were independently associated with the survival prognosis of patients with LUAD (Table III). However, in LUSC, although high LINC00628 expression was associated with worse overall survival (Fig. 3C; log-rank $\mathrm{P}=0.042$ ), the results of multivariate Cox regression analysis suggested that there was no statistical significance between LINC00628 expression and the prognosis of patients with LUSC $(\mathrm{HR}=1.896$; 95\% CI, 0.952-2.886; $\mathrm{P}=0.088)$. Nevertheless, TNM stage $(\mathrm{HR}=2.201$; 95\% CI, 1.417-3.071; $\mathrm{P}=0.013$ ) was an independent factor for the prognosis of patients with LUSC (Table IV).

\section{Discussion}

It has been reported that aberrant expression of lncRNA is associated with the progression of various cancer types (22-24). Several lncRNA molecules, such as lncRNA PTAR (25) and LINC01234 (26), have been reported to be abnormally expressed in NSCLC. In the present study, bioinformatics analysis of TCGA datasets demonstrated that the expression levels of LINC00628 were significantly upregulated in LUAD and LUSC tumor samples compared with normal tissue samples. In addition, analysis of our own clinical samples suggested that the expression levels of LINC00628 were significantly 
Table III. Cox regression analysis for patients with lung adenocarcinoma.

A, Univariate analysis

\begin{tabular}{lccr}
\hline Variables & HR & $95 \%$ CI & P-value \\
\hline Age & 1.331 & $0.727-1.992$ & 0.398 \\
Sex & 1.454 & $0.713-2.348$ & 0.551 \\
Smoking & 1.278 & $0.749-1.873$ & 0.412 \\
Tumor size & 1.496 & $0.888-2.256$ & 0.072 \\
Lymph node metastasis & 1.583 & $1.185-2.354$ & 0.037 \\
TNM stage & 2.639 & $1.798-4.551$ & 0.002 \\
LINC00628 expression & 2.949 & $1.982-5.173$ & $<0.001$ \\
\hline
\end{tabular}

$\mathrm{B}$, Multivariate analysis

\begin{tabular}{lccc}
\hline Variables & HR & $95 \%$ CI & P-value \\
\hline Age & 1.302 & $0.716-1.647$ & 0.549 \\
Sex & 1.465 & $0.730-2.232$ & 0.672 \\
Smoking & 1.272 & $0.746-1.808$ & 0.456 \\
Tumor size & 1.464 & $0.871-2.193$ & 0.108 \\
Lymph node metastasis & 1.532 & $1.035-2.159$ & 0.047 \\
TNM stage & 2.222 & $1.533-2.935$ & 0.006 \\
LINC00628 expression & 2.437 & $1.551-3.496$ & 0.001
\end{tabular}

LINC00628, long intergenic non-protein coding RNA 628; TNM,tumor-node-metastasis; HR, hazard ratio; CI, confidence interval.

increased in NSCLC, LUAD and LUSC tumor compared with adjacent normal tissue samples. In addition, the expression of LINC00628 was significantly associated with tumor size, histological type, lymph node metastasis and TNM stage in patients with NSCLC.

Furthermore, dysregulation of LINC00628 has been implicated in other malignancies $(27,28)$. For example, the overexpression of LINC00628 inhibits the proliferation, invasion and migration and promotes the apoptosis of osteosarcoma cells (27). LINC00628 expression is downregulated in the gastric cancer tissue and also suppresses gastric cancer cell proliferation and migration (28). The findings of the present study suggested that the expression levels of LINC00628 were significantly upregulated in LUAD and LUSC tissue samples, indicating that LINC00628 might be involved in tumor progression.

Increasing evidence indicates that lncRNA molecules may serve as potential biomarkers for NSCLC prognosis $(15,29)$. Schmidt et al (30) reported that MALAT-1 expression levels were associated with the survival of patients with NSCLC, and that this lncRNA acted as an oncogene. Additionally, $\mathrm{Lu}$ et al (31) validated LINC00673 as a novel oncogenic lncRNA and demonstrated the molecular mechanism by which it promotes NSCLC. In the present study, high LINC00628 was associated with worse overall survival in all patients with NSCLC, and LINC00628 expression could be used as the independent prognostic factor in LUAD.
Table IV. Cox regression analysis for patients with lung squamous cell carcinoma.

\begin{tabular}{lccc} 
A, Univariate analysis & & & \\
\hline Variables & HR & $95 \%$ CI & P-value \\
\hline Age & 1.158 & $0.781-1.683$ & 0.342 \\
Sex & 1.504 & $0.803-2.374$ & 0.261 \\
Smoking & 1.257 & $0.668-1.986$ & 0.552 \\
Tumor size & 1.334 & $0.923-1.894$ & 0.094 \\
Lymph node metastasis & 1.523 & $0.982-2.180$ & 0.061 \\
TNM stage & 2.473 & $1.652-3.385$ & 0.008 \\
LINC00628 expression & 1.998 & $0.986-3.165$ & 0.052
\end{tabular}

$\mathrm{B}$, Multivariate analysis

\begin{tabular}{lccc}
\hline Variables & HR & $95 \%$ CI & P-value \\
\hline Age & 1.126 & $0.776-1.676$ & 0.354 \\
Sex & 1.445 & $0.790-2.201$ & 0.277 \\
Smoking & 1.263 & $0.675-1.955$ & 0.531 \\
Tumor size & 1.265 & $0.879-1.746$ & 0.179 \\
Lymph node metastasis & 1.408 & $0.961-2.052$ & 0.074 \\
TNM stage & 2.201 & $1.417-3.071$ & 0.013 \\
LINC00628 expression & 1.896 & $0.952-2.886$ & 0.088
\end{tabular}

LINC00628, long intergenic non-protein coding RNA 628; TNM, tumor-node-metastasis; HR, hazard ratio; CI, confidence interval.

In a study by Navarro et al (32), the IncRNA HOTTIP was proposed as a prognostic biomarker in early-stage NSCLC, indicating the importance of IncRNAs for prognosis prediction in NSCLC. Although Xu et al (20) have suggested that LINC00628 was upregulated in LUAD tissue samples and promoted LUAD cell proliferation, migration and invasion, its expression and clinical significance remain unexplored in other subtypes of NSCLC. Our present study analyzed TCGA database data, and found that patients with LUAD and LUSC with high LINC00628 had a worse overall survival than those with low LINC00628 levels, which was consistent with the results obtained for patients with NSCLC. In the present study, survival analysis was carried out for patients with NSCLC, LUAD and LUSC separately. High LINC00628 was associated with worse overall survival in both LUAD and LUSC tumor tissue samples. However, multivariate Cox regression analysis indicated that LINC00628 expression was an independent prognostic factor for patients with LUAD, but not those with LUSC. Despite this result, the Kaplan-Meier survival curves generated our LUSC clinical samples indicated a significant difference in the survival of patients with low and high LINC00628 levels, which was at odds with the survival curves constructed using TCGA datasets. These paradoxical results may be mainly due to the differences in sample size and follow-up time between the two analyses. Indeed, the number of clinical samples was smaller than that of the TCGA 
datasets. Indeed, only 55 patients with LUSC were included in this study and collecting additional LUSC tissue samples for more precise statistical analysis may prove useful in future studies. In addition, the follow-up time in the present study was $\leq 60$ months, whereas the longest follow-up time for TCGA data was $>150$ months. In TCGA LUSC data, the survival rate appears to differ between the high and low-expression groups up to 100 months of patient follow-up. However, between 100 and 150 months, the survival of the low-expression group decreases at a faster rate than that of the high expression group, indicating that LINC00628 may be associated with early, but not late-stage survival. Therefore, it may be hypothesized that high LINC00628 expression was also associated with poor overall survival in LUAD. LINC00628 might represent a potential prognostic biomarker for patients with LUAD.

Moreover, ROC curve analysis indicated that LINC00628 had the ability to discriminate between LUAD and LUSC. Previous studies have identified multiple components of the immune system and molecules that might serve as diagnostic biomarkers for LUAD and $\operatorname{LUSC}(33,34)$. For instance, Shinmura et al (33) have suggested that chloride channel accessory 2 might be an immunohistochemical diagnostic biomarker for differentiating between primary LUSC and primary LUAD. In addition, fatty acids from erythrocyte total lipids might be used as diagnostic biomarkers of LUAD and LUSC, as demonstrated in the study by de Castro et al (34). Similarly, LINC00628 also has the potential to be a diagnostic biomarker for LUAD and LUSC.

There are some limitations to this study. As aforementioned, the limited sample size, especially for the LUSC samples (only 55 patients with LUSC), may have affected the accuracy of the results. Thus, additional LUSC tissue samples should be collected for more precise statistical analysis in subsequent studies. Moreover, in vitro experiments were not carried out, including analyses of the effects of LINC00628 on NSCLC cell proliferation, migration, invasion, to explore the different roles that LINC00628 might have in LUAD and LUSC based on the differential expression and clinical significance of LINC00628 in the two subtypes found in the present study. Thus, future studies will explore the biological function of LINC00628 using in vitro analyses. In addition, the NSCLC study population only contained LUAD and LUSC subtypes. and further analysis in other NSCLC subtypes is necessary.

In conclusion, LINC00628 expression levels were increased in NSCLC tumor tissue samples and associated with tumor development and survival prognosis. The elevated LINC00628 expression in LUAD compared with LUSC was independently associated with LUAD overall survival, but this relationship was not found in LUSC. Therefore, future studies should not only explore the biological function of LINC00628 in NSCLC, but also focus on the functional differences of LINC00628 in different NSCLC subtypes. The findings of the present study suggest that LINC00628 may become a diagnostic and prognostic biomarker for different subtypes of NSCLC.

\section{Acknowledgements}

Not applicable.

\section{Funding}

No funding was received.

\section{Availability of data and materials}

The datasets used and/or analyzed during the current study are available from the corresponding author on reasonable request.

\section{Authors' contributions}

TL conceived and designed the study. SX and XL analyzed and interpreted the data. TL and SX carried out the experiments. All authors wrote and revised the manuscript. TL and XL confirmed the authenticity of all the raw data. All authors read and approved the final manuscript.

\section{Ethics approval and consent to participate}

All experimental procedures were carried out in accordance with the guidelines of The Ethics Committee of Weifang People's Hospital and The Declaration of Helsinki. The present study was approved by The Ethics Committee of Weifang People's Hospital. Written informed consent was obtained from each patient.

\section{Patient consent for publication}

Not applicable.

\section{Competing interests}

The authors declare that they have no competing interests.

\section{References}

1. Ferlay J, Soerjomataram I, Dikshit R, Eser S, Mathers C, Rebelo M, Parkin DM, Forman D and Bray F: Cancer incidence and mortality worldwide: Sources, methods and major patterns in GLOBOCAN 2012. Int J Cancer 136: E359-E386, 2015.

2. Brown S, Banfill K, Aznar MC, Whitehurst P and Faivre Finn C: The evolving role of radiotherapy in non-small cell lung cancer. Br J Radiol 92: 20190524, 2019.

3. Yang Q, Tang Y, Tang C, Cong H, Wang X, Shen X and Ju S: Diminished LINC00173 expression induced miR-182-5p accumulation promotes cell proliferation, migration and apoptosis inhibition via AGER/NF- $\kappa$ B pathway in non-small-cell lung cancer. Am J Transl Res 11: 4248-4262, 2019.

4. Duma N, Santana-Davila R and Molina JR: Non-small cell lung cancer: Epidemiology, screening, diagnosis, and treatment. Mayo Clin Proc 94: 1623-1640, 2019.

5. Li N, Zhao J, Ma Y, Roy B, Liu R, Kristiansen K and Gao Q: Dissecting the expression landscape of mitochondrial genes in lung squamous cell carcinoma and lung adenocarcinoma. Oncol Lett 16: 3992-4000, 2018.

6. Faruki H, Mayhew GM, Serody JS, Hayes DN, Perou CM and Lai-Goldman M: Lung adenocarcinoma and squamous cell carcinoma gene expression subtypes demonstrate significant differences in tumor immune landscape. J Thorac Oncol 12: 943-953, 2017.

7. Tomasini P, Khobta N, Greillier L and Barlesi F: Ipilimumab: Its potential in non-small cell lung cancer. Ther Adv Med Oncol 4: 43-50, 2012.

8. Paez JG, Jänne PA, Lee JC, Tracy S, Greulich H, Gabriel S, Herman P, Kaye FJ, Lindeman N, Boggon TJ, et al: EGFR mutations in lung cancer: Correlation with clinical response to gefitinib therapy. Science 304: 1497-1500, 2004.

9. Mercer TR, Dinger ME and Mattick JS: Long non-coding RNAs: Insights into functions. Nat Rev Genet 10: 155-159, 2009. 
10. Wu Y, Liu H, Shi X, Yao Y, Yang W and Song Y: The long non-coding RNA HNF1A-AS1 regulates proliferation and metastasis in lung adenocarcinoma. Oncotarget 6: 9160-9172, 2015.

11. Chi Y, Wang D, Wang J, Yu W and Yang J: Long Non-Coding RNA in the pathogenesis of cancers. Cells 8: 1015, 2019.

12. Tasharrofi B and Ghafouri-Fard S: Long Non-coding RNAs as regulators of the mitogen-activated protein kinase (MAPK) pathway in cancer. Klin Onkol 31: 95-102, 2018.

13. Wei L, Yi Z, Guo K and Long X: Long noncoding RNA BCAR4 promotes glioma cell proliferation via EGFR/PI3K/AKT signaling pathway. J Cell Physiol 234: 23608-23617, 2019.

14. Su Y, Lu J, Chen X, Liang C, Luo P, Qin C and Zhang J: Long non-coding RNA HOTTIP affects renal cell carcinoma progression by regulating autophagy via the PI3K/Akt/Atg13 signaling pathway. J Cancer Res Clin Oncol 145: 573-588, 2019.

15. Ghafouri-Fard S, Abak A, Tondro Anamag F, Shoorei H, Majidpoor J and Taheri M: The emerging role of non-coding RNAs in the regulation of PI3K/AKT pathway in the carcinogenesis process. Biomed Pharmacother 137: 111279, 2021.

16. Chen DQ, Zheng XD, Cao Y, He XD, Nian WQ, Zeng XH and Liu XY: Long non-coding RNA LINC00628 suppresses the growth and metastasis and promotes cell apoptosis in breast cancer. Eur Rev Med Pharmacol Sci 21: 275-283, 2017.

17. Zhang MC, Zhang L, Zhang MQ, Yang GX, Wang FZ and Ding P: Downregulated LINC00628 aggravates the progression of colorectal cancer via inhibiting p57 level. Eur Rev Med Pharmacol Sci 24: 1763-1770, 2020.

18. Tian Y, Yu M, Sun L, Liu L, Wang J, Hui K, Nan Q, Nie X, Ren Y and Ren X: Distinct patterns of mRNA and lncRNA expression differences between lung squamous cell carcinoma and adenocarcinoma. J Comput Biol 27: 1067-1078, 2020.

19. Yu M, Tian Y, Wu M, Gao J, Wang Y, Liu F, Sheng S, Huo S and Bai J: A comparison of mRNA and circRNA expression between squamous cell carcinoma and adenocarcinoma of the lungs. Genet Mol Biol 43: e20200054, 2020.

20. Xu SF, Zheng Y, Zhang L, Wang P, Niu CM, Wu T, Tian Q, Yin XB, Shi SS, Zheng L and Gao LM: Long Non-coding RNA LINC00628 interacts epigenetically with the LAMA3 promoter and contributes to lung adenocarcinoma. Mol Ther Nucleic Acids 18: 166-182, 2019.

21. Livak KJ and Schmittgen TD: Analysis of relative gene expression data using real-time quantitative PCR and the 2(-Delta Delta C(T)) method. Methods 25: 402-408, 2001.

22. Hu S, Zheng Q, Xiong J, Wu H, Wang W and Zhou W: Long non-coding RNA MVIH promotes cell proliferation, migration, invasion through regulating multiple cancer-related pathways, and correlates with worse prognosis in pancreatic ductal adenocarcinomas. Am J Transl Res 12: 2118-2135, 2020.

23. Wang Y, Ding X, Hu H, He Y, Lu Z, Wu P, Tian L, Xia T, Yin J, Yuan $\mathrm{H}$, et al: Long non-coding RNA lnc-PCTST predicts prognosis through inhibiting progression of pancreatic cancer by downregulation of TACC-3. Int J Cancer 143: 3143-3154, 2018.

24. Feng J, Li J, Qie P, Li Z, Xu Y and Tian Z: Long non-coding RNA (lncRNA) PGM5P4-AS1 inhibits lung cancer progression by up-regulating leucine zipper tumor suppressor (LZTS3) through sponging microRNA miR-1275. Bioengineered 12: 196-207, 2021.
25. Yu W, Sun Z, Yang L, Han Y, Yue L, Deng L and Yao R: lncRNA PTAR promotes NSCLC cell proliferation, migration and invasion by sponging microRNA-101. Mol Med Rep 20: 4168-4174, 2019.

26. Chen Z, Chen X, Lei T, Gu Y, Gu J, Huang J, Lu B, Yuan L, Sun $\mathrm{M}$ and Wang Z: Integrative analysis of NSCLC identifies LINC01234 as an oncogenic lncRNA that interacts with HNRNPA2B1 and regulates miR-106b biogenesis. Mol Ther 28: 1479-1493, 2020.

27. He R, Wu JX, Zhang Y, Che H and Yang L: LncRNA LINC00628 overexpression inhibits the growth and invasion through regulating PI3K/Akt signaling pathway in osteosarcoma. Eur Rev Med Pharmacol Sci 22: 5857-5866, 2018.

28. Zhang ZZ, Zhao G, Zhuang C, Shen YY, Zhao WY, Xu J, Wang M, Wang CJ, Tu L, Cao H and Zhang ZG: Long non-coding RNA LINC00628 functions as a gastric cancer suppressor via long-range modulating the expression of cell cycle related genes. Sci Rep 6: 27435, 2016.

29. Song JY, Li XP, Qin XJ, Zhang JD, Zhao JY and Wang R: A fourteen-lncRNA risk score system for prognostic prediction of patients with non-small cell lung cancer. Cancer Biomark 29: 493-508, 2020.

30. Schmidt LH, Spieker T, Koschmieder S, Schäffers S, Humberg J, Jungen D, Bulk E, Hascher A, Wittmer D, Marra A, et al: The long noncoding MALAT-1 RNA indicates a poor prognosis in non-small cell lung cancer and induces migration and tumor growth. J Thorac Oncol 6: 1984-1992, 2011.

31. Lu W, Zhang H, Niu Y, Wu Y, Sun W, Li H, Kong J, Ding K, Shen $\mathrm{HM}, \mathrm{Wu} \mathrm{H}$, et al: Long non-coding RNA linc00673 regulated non-small cell lung cancer proliferation, migration, invasion and epithelial mesenchymal transition by sponging miR-150-5p. Mol Cancer 16: 118, 2017.

32. Navarro A, Moises J, Santasusagna S, Marrades RM, Viñolas N, Castellano JJ, Canals J, Muñoz C, Ramírez J, Molins L and Monzo M: Clinical significance of long non-coding RNA HOTTIP in early-stage non-small-cell lung cancer. BMC Pulm Med 19: 55, 2019.

33. Shinmura K, Igarashi $\mathrm{H}$, Kato $\mathrm{H}$, Kawanishi $\mathrm{Y}$, Inoue $\mathrm{Y}$, Nakamura S, Ogawa H, Yamashita T, Kawase A, Funai K and Sugimura H: CLCA2 as a novel immunohistochemical marker for differential diagnosis of squamous cell carcinoma from adenocarcinoma of the lung. Dis Markers 2014: 619273, 2014

34. de Castro J, Rodríguez MC, Martínez-Zorzano VS, Sánchez-Rodríguez P and Sánchez-Yagüe J: Erythrocyte fatty acids as potential biomarkers in the diagnosis of advanced lung adenocarcinoma, lung squamous cell carcinoma, and small cell lung cancer. Am J Clin Pathol 142: 111-120, 2014.

This work is licensed under a Creative Commons Attribution-NonCommercial-NoDerivatives 4.0 International (CC BY-NC-ND 4.0) License. 